\title{
Seasonality of birth and acute lymphoblastic leukemia
}

\author{
Tibor András Nyari, Pál Kajtár and Louise \\ Parker \\ Department of Child Health, University of Newcastle, \\ UK
}

Keywords: Acute lymphoblastic leukemia; infection in time of birth of children; infection in utero; prevention; seasonality.

Sir,

There is a growing body of evidence that exposure to infection is a risk factor for childhood leukemia. Recent epidemiological studies have suggested that environmental hazards (including infections), before or around birth, may also be associated with the risk of leukemia. In addition, a cyclic trend in occurrence could be interpreted as supportive evidence of exposure to infection.

Various methods of estimating and evaluating cyclic trends have been reported. The methodology for studying seasonal variation ranges from simple graphical means to more advanced and efficient techniques.

The aim of the current study was to investigate whether the seasonal trends of time of birth of children aged 0-4 years who were subsequently diagnosed with acute lymphoblastic leukemia (ALL).

Children born during 1981-1997 in South Hungary were evaluated. Data were available from the Central Demographic Agency on the number of births for each month over the study period. Registrations of first malignancies of children, born and diagnosed with ALL, under the age of five years in Hungary, before the end of 2002 were obtained from the Hungarian Pediatric Oncology Group.

Two methods for the detection of seasonal variations in epidemiological data were applied: Walter-Elwood's method and the logistic regression model including periodic functions (a sine and a cosine function, simultaneously). The magnitude of the seasonal variation as expressed by the amplitude of a simple harmonic oscil-

\footnotetext{
${ }^{*}$ Corresponding author:

Tibor András Nyari

University of Szeged

Department of Medical Informatics

H-6701, Szeged, P.O. Box: 427

Hungary

Tel./Fax: + 36-62-544-566

E-mail: nyari@dmi.szote.u-szeged.hu
}

lation was also calculated. The time at which the maximum and minimum incidences occurred was estimated using single (12-month period) and double (six-month period) peaks within the period of one year. Monthly unit was used to analyze the data. The pattern of annual cyclical variation was studied using date of birth. The $\chi^{2}$ goodness-of-fit test was used to examine the adequacy of the description of the data by a harmonic curve. Analyses were carried out using STATA (Version 8.0, StataCorp LP, College Station, Texas, USA) statistical packages.

The total number of childhood ALL cases diagnosed under the age of five for the study period was 121 . There were 481,984 live births in the study area, during the 17year interval of 1981-1997. The median age at diagnosis was 2.99 years (interquartile range [IQR]: 2.18-3.98 years). The median monthly incidence of ALL was 27.3 per 100,000 births (IQR: 19.16-30.58 per 100,000 births).

First, we tested whether there is a seasonal pattern with one maximum level and one minimum level per year. There was no evidence of seasonality of birth for ALL in children. However, both methods were applied to test further for seasonality with the six-month period. There was a cyclic pattern for ALL in all children diagnosed under the age of five years. Each method demonstrated two peaks, one in early February and a second in early August, and two troughs in May and November (amplitude 0.34, $\mathrm{P}=0.027$ ).

Seasonal variation related to time of birth would indicate exposure to an infection in utero or around the time of birth. We found evidence of seasonality related to month of birth with peaks in February and August. Both peaks could reflect the seasonality of infectious diseases in temperate climates: respiratory virus infections (e.g., influenza) show marked seasonality occurring in the winter months, and gastrointestinal infections peak in the summer months.

Although several date of birth studies reported marked and significant evidence of seasonality, there was variability in the time of a single peak incidence. A February peak was reported by Feltbower et al. [1] in Northern England, UK, who studied children diagnosed with ALL aged 1-6 years, and by Higgins et al. [2] in a study of 15,835 cases of childhood leukemia born and diagnosed in the UK between 1953-95 and diagnosed before1960. A summer peak was found by Ross and co-workers [3] in the USA. ALL also demonstrated statistically significant monthly variation in the date of birth (peak in October) in an Iranian study. 
Because our study was designed specifically to investigate the role of exposure to infection during prenatal life and around the time of birth, it was able to address the potential role of infection as a general risk factor for acute lymphoblastic leukemia in young children as postulated by Greaves.

In our study the population denominator was accurately known providing a large, representative sample of Hungarian children over a 17 -year period. In addition, the observed association is consistent with other epidemiological evidence of a potential role of infections in the etiology of ALL.

This study was supported by Hungarian Eötvös fellowship of the Hungarian Scholarship Board and Bolyai fellowship of the Hungarian Academy of Sciences.

\section{References}

[1] Feltbower RG, MS Pearce, HO Dickinson, L Parker, PA McKinney: Seasonality of birth for cancer in Northern England, UK. Paediatr Perinat Epidemiol 15 (2001) 338

[2] Higgins CD, I dos-Santos-Silva, CA Stiller, AJ Swerdlow: Season of birth and diagnosis of children with leukaemia: an analysis of over 15000 UK cases occurring from 1953-95. Br J Cancer 84 (2001) 406

[3] Ross JA, RK Severson, AR Swensen, BH Pollock, JG Gurney, LL Robison: Seasonal variations in the diagnosis of childhood cancer in the United States. $\mathrm{Br} \mathrm{J}$ Cancer 81 (1999) 549

Received February 9, 2006. Revised August 9, 2006. Accepted September 11, 2006. 